\title{
Associations among Genetic Variants and Intracranial Aneurysm in a Chinese Population
}

\author{
Bingyang $\mathrm{Li}^{1 *}$, Chongyu Hu${ }^{2 *}$, Junyu $\mathrm{Liu}^{3}$, Xin $\mathrm{Liao}^{1}$, Jiayu Xun ${ }^{1}$, Manqian Xiao ${ }^{1}$, and Junxia Yan \\ ${ }^{1}$ Department of Epidemiology and Health Statistics, XiangYa School of Public Health, Central South University, Hunan; \\ ${ }^{2}$ Department of Neurology, Hunan People's Hospital, Changsha; \\ ${ }^{3}$ Department of Neurosurgery, XiangYa Hospital, Central South University, Changsha, Hunan, China.
}

Purpose: Genome-wide association studies (GWAS) have revealed that common variants on or near EDNRA, HDAC9, SOX17, $R P 1, C D K N 2 B-A S 1$, and $R B B P 8$ genes are associated with intracranial aneurysm (IA) in European or Japanese populations. However, due to population heterogeneity, whether these loci are associated with IA pathogenesis in Chinese individuals is still unknown. The purpose of this study was to investigate associations among GWAS-identified loci and risk of IA in a Chinese population. Materials and Methods: A total of 765 individuals (including 230 IA patients and 535 controls) were involved in this study. Twelve single nucleotide polymorphisms (SNPs) of candidate loci were genotyped using the Sequenom MassARRAY platform. Associations were analyzed using univariate or multivariate logistic regression analysis.

Results: SNPs in CDKN2B-AS1 (especially rs10757272) showed significant associations with IA in dominant and additive models [odds ratio (OR), 2.99 and 1.43; 95\% confidence interval (CI), 1.44-6.24 and 1.10-1.86, respectively]. A SNP near HDAC9(rs10230207) was associated with IA in the dominant model (OR, 1.42; 95\% CI, 1.01-1.99). One SNP near RP1 (rs1072737) showed a protective effect on IA in the dominant model (OR, 0.66; 95\% CI, 0.46-0.95), while another SNP in $R P 1$ (rs9298506) showed a risk effect on IA in a recessive model (OR, 3.82; 95\% CI, 1.84-7.91). No associations were observed among common variants near EDNRA, SOX17, or RBBP8 and IA.

Conclusion: These data partially confirmed earlier results and showed that variants in $C D K N 2 B-A S 1, R P 1$, and $H D A C 9$ could be genetic susceptibility factors for IA in a Chinese population.

Key Words: Intracranial aneurysm, susceptibility genes, genetic polymorphisms

\section{INTRODUCTION}

Intracranial aneurysm (IA) is a complex disease characterized by pathologic dilatations of the cerebral arteries. The prevalence of IA is $2-5 \%$ worldwide and approximately $7 \%$ in Chinese adults aged 35 to 75 years old. ${ }^{1,2}$ The rupture of an IA

Received: December 19, 2018 Revised: April 2, 2019

Accepted: April 24, 2019

Corresponding author: Junxia Yan, PhD, Department of Epidemiology and Health Statistics, XiangYa School of Public Health, Central South University, Shang Mayuanling, KaiFu District, Changsha, 410078, China.

Tel: 86-0731-84805465, Fax: 86-0731-84805454,E-mail: 20457456@qq.com

*Bingyang Li and Chongyu Hu contributed equally to this work.

- The authors have no potential conflicts of interest to disclose.

(C) Copyright: Yonsei University College of Medicine 2019

This is an Open Access article distributed under the terms of the Creative Commons Attribution Non-Commercial License (https://creativecommons.org/licenses/ by-nc/4.0) which permits unrestricted non-commercial use, distribution, and reproduction in any medium, provided the original work is properly cited. leads to subarachnoid hemorrhage (SAH), which is one of the most devastating known neurological conditions. ${ }^{3}$ Although the pathogenesis of IA has been explored for many years, the mechanisms of its formation, growth, and rupture remain largely unknown. IA treatment mainly relies on invasive neurosurgical clipping or endovascular coiling with a risk of procedural morbidity and mortality. ${ }^{4}$

Multiple factors, such as hypertension, smoking, excessive alcohol consumption, increasing age, and female sex, are known to be risk factors for IA. ${ }^{5}$ Furthermore, the familial occurrence of IA and its association with certain genetic disorders, such as autosomal dominant polycystic kidney disease and Ehlers-Danlos syndrome, suggest a genetic component important to IA formation. ${ }^{6}$ Upon linkage analysis and candidate gene association studies, several common variants of collagen type I alpha 2 chain (COL1A2: rs42524), collagen type III alpha 1 chain (COL3A1: rs1800255), heparan sulfate proteo- 
glycan 2 (HSPG2: rs3767137), serpin family A member 3 (SERPINA3: rs4934), and versican (VCAN: rs251124 and rs173686) were found to be associated with IA. ${ }^{7}$ Further genome-wide association studies (GWAS) and case-control replication analysis identified novel common variants for IA, such as variants near or on endothelin receptor type A (EDNRA: rs6841581), histone deacetylase 9 (HDAC9: rs6841581), sex determining region Y-box 17 (SOX17: rs10958409), RP1 axonemal microtubule associated ( $R P 1$ : rs9298506), CDKN2B antisense RNA 1 (CDKN2B-AS1: rs10757278), and RB binding protein 8, endonuclease (RBBP8: rs11661542) ${ }^{8-11}$ Despite limited effect sizes of the identified variants [odds ratio (OR) 1.1-1.5], these results have provided the foundation for future studies.

Notwithstanding, the GWAS and subsequent replication studies were mainly conducted in Dutch, Finnish, Japanese, and Korean populations. While a few studies have explored associations between $E D N R A$ and $C D K N 2 B-A S 1$ loci and IA in Chinese populations, due to the small sample sizes and limited statistical power, no definite conclusions were reached in regards to whether these loci contribute to IA pathogenesis in the Chinese population or not. ${ }^{12-15}$ Thus, the aim of the present study was to investigate whether there are associations between identified loci and IA risk in a Chinese population.

\section{MATERIALS AND METHODS}

\section{Study population}

In cooperation with hospitals in Hunan Province of China, we recruited IA patients from the Department of Neurology and Neurosurgery of Xiangya Hospital and Hunan People's Hospital from December 2016 to February 2018. All patients with IA who agreed to participant in this study during the study period were enrolled. The cases were diagnosed by magnetic resonance imaging, computed tomography angiography, or digital subtraction angiography. Individual information and lifestyle data were collected by interview. Control subjects were recruited from the same geographic region who attended a community routine annual health check-up during the same period. The control subjects met the following criteria: 1) no medical history of any vascular diseases, including IA or SAH, and 2) no family history of IA or SAH in first-degree relatives. Peripheral blood samples were obtained from all enrolled individuals. This study was approved by the Medical Ethics Committee of Clinical Pharmacology Institute, Central South University (CTXY-150002-1), and written informed consent was obtained from all participants.

\section{Single nucleotide polymorphism selection and genotyping}

Based on previous studies, 12 single nucleotide polymorphisms (SNPs) were selected by searching for SNPs in the GWAS that showed significant associations with IA., ${ }^{9,10,15-18}$ Genomic DNA was extracted from peripheral blood leukocytes using TIANamp Blood DNA Extraction Kits (TIANGEN Biotech Co., Ltd., Beijing, China). The DNA samples were stored at $-80^{\circ} \mathrm{C}$ until ready for use. SNPs were genotyped using the MassARRAY iPlex platform (Agena Bioscience Inc., San Diego, CA, USA). Primers were designed using ASSAY DESIGN SUITE V2.0 based on a SNP locus (http://agenacx.com, Agena Bioscience Inc.) (Table 1). The PCR reaction comprised of $1 \mu \mathrm{L}$ of genomic DNA (20-50 ng) and $4 \mu \mathrm{L}$ of Multiplex PCR mixture that contained $1.8 \mu \mathrm{L}$ of $\mathrm{dddH}_{2} \mathrm{O}, 0.5 \mu \mathrm{L}$ of $10 \times$ PCR buffer, $0.4 \mu \mathrm{L}$ of $\mathrm{MgCl}_{2}(25 \mathrm{mM}), 0.1 \mu \mathrm{L}$ of dNTP $(25 \mathrm{mM}), 0.2 \mu \mathrm{L}$ of HotstarTaq ( $5 \mathrm{U} / \mu \mathrm{L}$, Agena Bioscience Inc.), and $1 \mu \mathrm{L}$ of PCR Primer mix. The PCR conditions were as follows: initial denaturing at $95^{\circ} \mathrm{C}$ for 2 minutes, followed by 45 cycles of denaturing at $95^{\circ} \mathrm{C}$ for 30 seconds, annealing at $56^{\circ} \mathrm{C}$ for 30 seconds, and final extension at $72^{\circ} \mathrm{C}$ for 60 seconds. The last step was to hold at $25^{\circ} \mathrm{C}$ indefinitely. After shrimp alkaline phosphatase reaction, single base extension, and resin desalting reaction, SNP alleles were identified by different masses of the extended prime primers using matrix-assisted laser desorption/ionization-time of flight (MALDI-TOF) mass spectrometry. The mass spectrometry peaks were detected using Typer 4.0 software (Agena Bioscience Inc.) and the genotypes of each sample target locus were interpreted based on the mass spectrum peak map. Genotyping was performed by an operator blinded to the sample status (case or control subjects).

\section{Statistical analysis}

General clinical features of the case and control groups are described as a mean \pm standard deviation (SD) and were compared using Student's t-test for continuous variables. Categorical variables are presented as proportions and were compared using the chi-square test or Fisher's exact test. The Hardy-Weinberg equilibrium of control subjects was tested using Stata 11.2 (StataCorp LLC, College Station, TX, USA). Pair-wise linkage disequilibrium (LD) was estimated using Haploview v.4.2 (https://www.broadinstitute.org/haploview/haploview). ${ }^{19}$ Pairwise $\mathrm{LD}$ parameters $\mathrm{r}^{2}$ and $\mathrm{D}^{\prime}$ were used as criteria for judging $\mathrm{LD}^{20}$ If $\mathrm{r}^{2} \geq 80 \%$, SNPs were considered in strong LD. Meanwhile, if $\mathrm{D}^{\prime}=1, \mathrm{SNP}$ s were considered in complete $\mathrm{LD}$. Univariate and multivariate logistic regression analysis were used to estimate ORs and 95\% confidence intervals (CI) of each SNP. A $p$ value less than 0.05 was considered statistically significant. Statistical analyses were performed using SPSS 21.0 software (IBM Corp., Armonk, NY, USA).

\section{RESULTS}

\section{Characteristics of study population}

This study included 230 IA patients and 535 normal individuals without known IA or other cerebrovascular diseases. The demographic and clinical characteristics of the patients and 
Bingyang Li, et al.

Table 1. The Positions and Primers Designed for the 12 SNPs

\begin{tabular}{|c|c|c|c|c|c|}
\hline $\operatorname{SNP}(D / d)^{*}$ & Position $^{\dagger}$ & Length (bp) & Gene & Primer & Primer sequence \\
\hline \multirow{2}{*}{ rs6841581(G/A) } & \multirow{2}{*}{ chr4:147480038 } & \multirow{2}{*}{113} & \multirow{2}{*}{$\operatorname{EDNRA}(2 \mathrm{~kb})^{\ddagger}$} & Forward & 5'-ACATTCACTCTTGGATCCCC-3' \\
\hline & & & & Reverse & 5'-TGCTTGGTGTTGAGTGTTAG-3' \\
\hline \multirow{2}{*}{ rs10230207(G/T) } & \multirow{2}{*}{ chr7:19571684 } & \multirow{2}{*}{124} & \multirow{2}{*}{$H D A C 9(569 \mathrm{~kb})^{\ddagger}$} & Forward & 5'-GTCTAAAACACACTGTCCCC-3' \\
\hline & & & & Reverse & 5'-ACTGGCTTTCCCAAAGGAAC-3' \\
\hline \multirow{2}{*}{ rs1504749(A/C) } & \multirow{2}{*}{ chr8:54398151 } & \multirow{2}{*}{121} & \multirow{2}{*}{$\operatorname{SOX17}(60 \mathrm{~kb})^{\ddagger}$} & Forward & 5’-TGTCCTGCCCTCCGAAAAAG-3’ \\
\hline & & & & Reverse & 5'-AGGTCTTGGCTGGTATTAAC-3' \\
\hline \multirow{2}{*}{ rs10958409(G/A) } & \multirow{2}{*}{ chr8:54414531 } & \multirow{2}{*}{120} & \multirow{2}{*}{ SOX17(43 kb $)^{\ddagger}$} & Forward & 5'-GCAGATGGCAGTTAAGGAAG-3' \\
\hline & & & & Reverse & 5'-TAGTGATTGCCCATCAGAGG-3' \\
\hline \multirow{2}{*}{ rs1072737(T/G) } & \multirow{2}{*}{ chr8:54501764 } & \multirow{2}{*}{129} & \multirow{2}{*}{$R P 1(8 \mathrm{~kb})^{\ddagger}$} & Forward & 5'-TTCTTCTTCAGGGACTCCAG-3' \\
\hline & & & & Reverse & 5'-TGTGGCAATGAAAACTACCC-3' \\
\hline \multirow{2}{*}{ rs9298506(A/G) } & \multirow{2}{*}{ chr8:54524964 } & \multirow{2}{*}{120} & \multirow{2}{*}{$R P 1$} & Forward & 5'-GACTTAAGGGTGTCTGGAAG-3' \\
\hline & & & & Reverse & 5'-GGAAACAGCAAACAGCCTAC-3' \\
\hline \multirow{2}{*}{ rs1333040(C/T) } & \multirow{2}{*}{ chr9:22083405 } & \multirow{2}{*}{110} & \multirow{2}{*}{ CDKN2B-AS1 } & Forward & 5'-TTTGGGAGCCACTGTTGTAG-3' \\
\hline & & & & Reverse & 5'-CATTCAAGAGAGACAGGAGG-3' \\
\hline \multirow{2}{*}{ rs10757272(C/T) } & \multirow{2}{*}{ chr9:22088261 } & \multirow{2}{*}{109} & \multirow{2}{*}{ CDKN2B-AS1 } & Forward & 5'-GCTGGGATTATGAAAGGTCG-3' \\
\hline & & & & Reverse & 5'-AATACCACTGACATGGAGAG-3' \\
\hline \multirow{2}{*}{ rs1333042(A/G) } & \multirow{2}{*}{ chr9:22103814 } & & & Forward & 5'-TTTGAGTAGACAGCCAACCC-3' \\
\hline & & 123 & CDKINZB-ASI & Reverse & 5'-TTCAATAGCCAGAAAACCCC-3' \\
\hline rs10733376/G/Cl & chro.22114470 & & & Forward & 5'-CTTTGCСTTCСТCTCCTTTC-3' \\
\hline ISTU/333/0(u/C) & chry:221144/0 & 118 & CDKIVZB-ASI & Reverse & 5'-ATTCGCCGAGGTAAACCTTG-3' \\
\hline rs10757278(A/G) & & & CDKN2B-AS1 $(3 \mathrm{~kb})^{\ddagger}$ & Forward & 5'-ACTCTGTCTTGATTCTGCAT-3' \\
\hline ISIU/J/L/O(A/G) & cnry:ZZ1Z44/8 & 90 & CDKIVZB-AS/(J KD) & Reverse & 5'-AGTTGGAACTGAACTGAGGC-3' \\
\hline & & & RBВP8 $(270 \mathrm{~kb})^{\ddagger}$ & Forward & 5'-AGCTGGACCATGCCATTGTG-3' \\
\hline 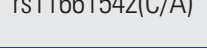 & cnrTo:ZZO43/3L & 115 & RВВPO (Z/U KD) & Reverse & 5'-ACCCACAACAATTAGTTCCC-3' \\
\hline
\end{tabular}

SNP, single nucleotide polymorphism; kb, kilobase.

${ }^{*}$ Allele D indicates wild-type alleles; d, risk alleles, ${ }^{\dagger}$ The position in Genome Reference Consortium Human genome build 38.p12 (GRCh38.p12), ${ }^{\ddagger}$ SNP near the gene (distance).

Table 2. Demographic and Clinical Characteristics of the Study Participants

\begin{tabular}{lccc}
\hline \multicolumn{1}{c}{ Variables } & $\begin{array}{c}\text { Case } \\
(\mathbf{n}=\mathbf{2 3 0 )}\end{array}$ & $\begin{array}{c}\text { Control } \\
(\mathbf{n = 5 3 5 )}\end{array}$ & $\boldsymbol{p}$ value \\
\hline Mean age \pm SD (yr) & $57.0 \pm 10.7$ & $64.7 \pm 10.9$ & 0.000 \\
\hline Female, $\mathrm{n}(\%)$ & $142(61.7)$ & $340(63.6)$ & 0.634 \\
\hline Hypertension, $\mathrm{n}(\%)$ & $128(55.7)$ & $296(55.3)$ & 0.934 \\
Smoking, $\mathrm{n}(\%)$ & $45(19.6)$ & $147(27.5)$ & 0.021 \\
\hline Alcohol consumption, $\mathrm{n}(\%)$ & $36(15.7)$ & $108(20.2)$ & 0.141 \\
\hline Single intracranial aneurysm, $\mathrm{n}(\%)$ & $167(72.6)$ & - & \\
Site of intracranial aneurysm, $\mathrm{n}(\%)$ & & & \\
\hline Internal carotid artery & $150(47.6)$ & - & \\
\hline Middle cerebral artery & $57(18.1)$ & - & \\
\hline Anterior communicating artery & $41(13.0)$ & - & \\
\hline Anterior cerebral artery & $19(6.0)$ & - & \\
\hline Posterior communicating artery & $15(4.8)$ & - & \\
\hline Vertebral artery & $9(2.9)$ & - & \\
\hline Basilar artery & $8(2.5)$ & - & \\
\hline Posterior cerebral artery & $4(1.3)$ & - & \\
\hline Other & $12(3.8)$ & - & \\
\hline
\end{tabular}

SD, standard deviation. controls are summarized in Table 2. In short, female sex was predominant in both groups. The mean age of the controls was older than that of the patients, and smokers were more frequent in the control group. There were no significant distribution differences in sex, hypertension, and alcohol consumption status between case and control groups. In the case group, $72.6 \%$ individuals had a single aneurysm. The aneurysms were primarily located in the internal carotid artery $(47.6 \%)$, followed by the middle cerebral artery (18.1\%), anterior communicating artery (13\%), and other locations.

\section{Hardy-Weinberg equilibrium and linkage disequilibrium analysis}

All SNPs showed $100 \%$ genotyping call rate, and the risk allele frequencies were more than $10 \%$ in the participants. The genotype distributions of all SNPs were in Hardy-Weinberg equilibrium in the healthy controls ( $p>0.05)$ (Table 3$)$. LD analysis showed that the SNPs of SOX17 (rs1504749 and rs10958409) and CDKN2B-AS1 (rs10757272 and rs1333042) were in strong LD $\left(\mathrm{r}^{2}>0.8\right)$ (Fig. 1). We chose to focus on SNPs rs10958409 and rs10757272. 
Table 3. Multivariate Logistic Regression Analysis of Associations among the 12 SNPs and Risk of Intracranial Aneurysm in a Chinese Population

\begin{tabular}{|c|c|c|c|c|c|c|c|c|c|c|}
\hline \multirow{2}{*}{ SNP } & \multirow{2}{*}{ ene } & \multicolumn{2}{|c|}{ Genotype* } & \multirow{2}{*}{$\begin{array}{c}p^{\dagger} \\
\text { HWE }\end{array}$} & \multicolumn{2}{|c|}{ Dominant model } & \multicolumn{2}{|c|}{ Recessive model } & \multicolumn{2}{|c|}{ Additive model } \\
\hline & & Case (n) & Control (n) & & OR (95\% Cl) & $p$ & OR (95\% Cl) & $p$ & OR (95\% CI) & $p$ \\
\hline 041001 & EDNRA & $142 / 78 / 10$ & $298 / 202$ & 0.922 & 8210 & 0.236 & 44) & 0.295 & $-1.09)$ & 168 \\
\hline 0230207 & HDAC9 & 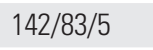 & 369 & 880 & & & & & 69) & \\
\hline & SOX1 & ?ח & & & & & & & & \\
\hline & SOX17 & & & 454 & & 0.728 & & 0.084 & & 0.634 \\
\hline & $R P 1$ & 8/5-17 & & 0.251 & & 0.024 & & 0.771 & & 0.060 \\
\hline 298506 & $R P 1$ & 1 & & .213 & & 0.6 & & 0.000 & & 0.087 \\
\hline 33040 & CDKI & $7 / 97 / 1$ & 48 & & & & & 19 & & 0 \\
\hline 57272 & CDKI & 10 & & 0.50 & & 0.0 & & 0.082 & & 0.008 \\
\hline 33042 & CDKI & //100/120 & 50 & 0.318 & & 0.003 & & 0.338 & .71) & 0.037 \\
\hline rs10733376 & CDKN2B-AS1 & 14/125/91 & $72 / 257 / 206$ & 0.562 & $2.89(1.52-5.49)$ & 0.001 & $1.02(0.73-1.42)$ & 0.922 & $1.24(0.96-1.60)$ & 0.096 \\
\hline rs10757278 & CDKN2B & $36 / 126 / 68$ & $122 / 280 /$ & 0.27 & & 0.0 & & 0.317 & $1.27(0.9$ & 0.054 \\
\hline rs11661542 & RBBP8 & $68 / 108 / 54$ & 146/276/113 & 0.409 & $0.86(0.60-1.23)$ & 0.394 & $1.08(0.73-1.59)$ & 0.713 & $0.96(0.76-1.21)$ & 0.744 \\
\hline
\end{tabular}

SNP, single nucleotide polymorphism; $\mathrm{OR}$, odds ratio; $\mathrm{Cl}$, confidence interval.

${ }^{*}$ Genotype presented as wild type/heterozygous/homozygous, ${ }^{\dagger}$ Hardy-Weinberg equilibrium (HWE) $p$ value for the control group.

\section{Associations between candidate loci and IA}

The genotypes of 12 SNPs and their associations with IA are presented in Table 3. SNPs in CDKN2B-AS1 (especially rs10757272) showed significant associations with IA in univariate logistic regression analysis in different genetic models (Supplementary Table 1, only online). These associations between $C D$ $K N 2 B-A S 1$ variations and IA remained significant after adjustment for age and smoking in the dominant and additive models (Table 3). One SNP in RP1 (rs9298506) was markedly associated with IA in a recessive model (OR, 3.82; 95\% CI, 1.84-7.91). However, another SNP near RP1 (rs1072737) showed a protective effect on IA in a dominant model (OR, 0.66; 95\% CI, 0.460.95 ) both in univariate and multivariate analyses. The above associations were verified in the 208 Chinese Han individuals in the 1000 Genomes Project as additional controls (Supplementary Table 2, only online). The SNP near HDAC9(rs10230207) showed conflicting results with IA. It was associated with IA in the dominant model when adjusted for age and smoking (OR, 1.42; 95\% CI, 1.01-1.99), while this association was not replicated in the additional control (Table 3, Supplementary Tables 1 and 2, only online). SNPs near EDNRA (rs6841581), SOX17 (rs1504749 and rs10958409), and RBBP8 (rs11661542) did not show associations with IA in any genetic model, whether adjusted age and smoking or not (all $p>0.05$ ).

\section{Comparison of the associations with other studies}

The comparisons of our results with those of other studies are summarized in Supplementary Table 3 (only online). Generally, only $C D K N 2 B-A S 1$ loci showed similar associations in Chinese population with those in European and Japanese populations. For the associations of common variants on or near EDNRA, HDAC9, SOX17, PR1, and RBBP8 loci and IA, inconsistent results were observed in different populations.

\section{DISCUSSION}

In this study, we investigated associations among 12 SNPs in loci identified in a previous GWAS and the risk of IA in a Chinese population. The results confirmed that genetic variants in $C D K N 2 B-A S 1, R P 1$, and HDAC9 loci were significantly associated with the risk of IA in a Chinese population. However, the associations of variants on or near EDNRA, SOX17, and $R B B P 8$ loci with IA were not replicated, suggesting that there were both common and specific susceptibility factors in the Chinese population.

$C D K N 2 B-A S 1$, also known as $A N R I L$, is located within the $C D K N 2 B-C D K N 2 A$ gene cluster at chromosome 9p21.3. ${ }^{17}$ SNPs on this locus, especially rs10757272, have been identified to be associated with various vascular diseases, including coronary artery diseases, stroke, myocardial infarction, and abdominal aortic aneurysm. ${ }^{21}$ Multiple studies also found that common variants of this locus significantly increased the risk of IA in European and Japanese populations, indicating that the $C D$ $K N 2 B-A S 1$ locus might contain a common genetic risk factor(s) for multiple vascular diseases..$^{10,14,17,18,21-25}$ In agreement with the previous findings, we found that five genotyped SNPs (rs1333040, rs10757272, rs1333042, rs10733376, and rs10757278) on or near CDKN2B-AS1 were significantly associated with IA in a dominant model and that rs10757272 was further associated with IA in an additive model in Chinese population. Although the exact roles of $C D K N 2 B-A S 1$ variants and IA risk are not clear, an animal study revealed that targeted deletion of $9 \mathrm{p} 21$ risk loci significantly attenuated the expression of two CDKN2BASs' neighboring tumor-suppressor genes $\left(p 15^{I N K 4 b}\right.$ and $\left.p 16^{I N K 4 a}\right)$, thereby altering coronary artery disease progression. ${ }^{26} P 15^{I N K 4 b}$ and $p 16^{I N K 4 a}$ have been found to play roles in regulating cell cycle and promoting vascular remodeling. ${ }^{27}$ Variants of $C D$ $K N 2 B-A S 1$ have been shown to participate in the formation of 


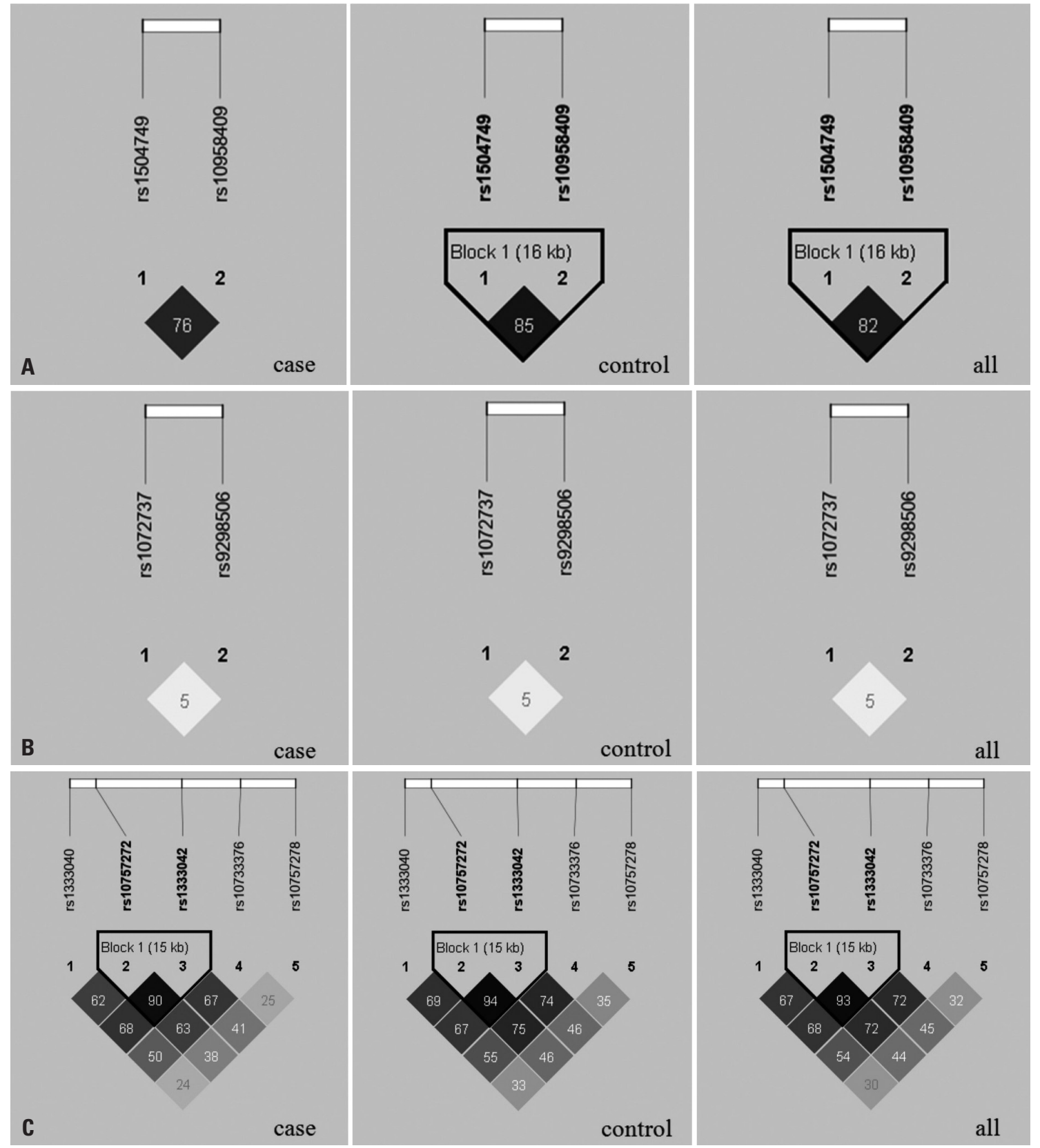

Fig. 1. Linkage disequilibriums of $S O X 17(\mathrm{~A}), R P 1(\mathrm{~B})$ and $C D K N 2 B-A S 1(\mathrm{C})$ in case, control, and total groups.

atherosclerosis by inhibiting the expression of $p 15^{I N K 4 b}$ and $p 16^{I N K 4 a} \cdot{ }^{28}$ Thus, we speculate that individuals with $C D K N 2 B-$ $A S 1$ variants may be prone to IA due to reduced expression of $p 15^{I N K 4 b}$ and $p 16^{I N K 4 a}$. This should be explored in future experimental studies.

$R P 1$ is located at chromosome $8 \mathrm{q} 11.23-\mathrm{q} 12.1$. Variants in this gene were reported to affect retinal phenotype. ${ }^{29}$ Previous GWASs reported that rs1072737 and rs9298506 were near 3'SOX17. ${ }^{10,15}$ However, according to the most recent Genome Reference Consortium human genome build 38 (GRCh38.p12), rs9298506 is located on the intron of $R P 1$, and rs1072737 is located $8 \mathrm{~kb}$ upstream from $R P 1$, but $41 \mathrm{~kb}$ downstream from
$S O X 17$, which is much closer to $R P 1$. Therefore, in this study, we classified rs1072737 and rs9298506 into RP1. The two SNPs of $R P 1$ lacked strong LD both in our study and two other populations (East Asian and European) in the 1000 Genomes Project data (Fig. 1, Supplementary Fig. 1, only online). Thus these SNPs may have different directions of effect on IA. For rs1072737, Foroud, et al. ${ }^{16}$ found that the $\mathrm{G}$ allele of it increased the risk of IA in individuals of European-ancestry in an additive model. However, Hong, et al. ${ }^{30}$ reported that the G allele of rs1072737 had a protective effect on IA in a Korean population in an allelic model. For rs9298506, Yasuno, et al. ${ }^{10}$ and Bilguvar, et al. ${ }^{15}$ reported that the $\mathrm{G}$ allele had a protective effect on IA in Euro- 
pean and Japanese populations in an additive model. Nevertheless, in this study, we found that rs1072737 showed a protective effect against IA in a dominant model, while rs9298506 showed a risk effect for IA in a recessive model. Furthermore, the same direction effect of associations between these two SNPs and IA were replicated when we further used data from 208 Chinese Han individuals from the 1000 Genome Project as controls (Supplementary Table 2, only online). This provided further evidence to support that $R P 1$ could be a potential susceptibility gene for IA in Chinese. Due to genetic heterogeneity, common variants of $R P 1$ might affect IA in different genetic models of diverse ethnic populations. Previous functional analysis of $R P 1$ mainly focused on its role for retinal disease, ${ }^{29}$ and the full-length nature of variants in $R P 1$ is still unknown. Accordingly, the mechanisms of $R P 1$ variants affecting IA need to be further explored.

HDAC9 is located at chromosome $7 \mathrm{p} 21.1$ and plays roles in regulating ox-LDL-induced endothelial cell apoptosis and inflammatory factor expression. ${ }^{31}$ Previous studies have shown that chromosome 7p21.1 and HDAC9 genetic variants were associated with ischemic stroke in Chinese Han, European, and Caucasians populations. ${ }^{32-34}$ In a subsequent GWAS, Foroud, et al. ${ }^{17}$ found that rs10230207 near HDAC9 had a risk effect on IA in white and Dutch populations. Consistent with the findings of Foroud's study, we found that rs 10230207 increased the risk of IA in our Chinese population after adjustment for age and smoking. Given the small effect size of $H D A C 9$, the exact association between HDAC9 and IA might be covered by other environmental factors. A recent study found that there were significantly gene expression differences between HDAC9 risk allele-positive and negative large vessel atherosclerotic stroke patients, which illustrated that HDAC9 variants may be associated with peripheral immune, lipid, and clotting systems. ${ }^{35}$ Whether HDAC9 variants affect IA in a similar manner with that in large vessel atherosclerotic stroke is still unknown. Exact mechanisms should be studied in the future.

EDNRA is located in chromosome 4q31.22-q31.23 and encodes the receptor for endothelin-1, which is a peptide that plays a role in potent and long-lasting vasoconstriction..$^{36} \mathrm{An}$ allele of rs6841581 near EDNRA showed a protective effect on IA in European, Japanese, and Korean populations in an additive model..$^{9,37}$ However, in the present study, we found that rs6841581 was not associated with IA in any genetic models of our Chinese population. The result was consistent with a previous study by Liu, et al. ${ }^{12}$ Given that genetic heterogeneity of IA exists in different populations, rs6841581 near EDNRA may be not evoke susceptibility to IA in Chinese.

SOX17 is located at chromosome 8q and encodes a member of the SRY-related HMG-box family of transcription factors involved in the regulation of embryonic development and in the determination of the cell fate..$^{38}$ Bilguvar, et al. ${ }^{15}$ reported that rs10958409 near SOX17 provided a risk effect on IA in a Japanese population in an additive model. However, the asso- ciation was not able to be replicated in subsequent studies. Hashikata, et al. ${ }^{11}$ and Yasuno, et al. ${ }^{10}$ reported that rs10958409 was not associated with IA in an independent Japanese population. In a European population, rs10958409 increased the risk of IA in an additive model, ${ }^{15}$ while in a Korean population, rs10958409 was not associated with IA in allelic and additive models. ${ }^{30,37}$ Consistent with the result in the Korean population, we did not identify any associations between rs10958409 and IA in different genetic models. The LD structure of SOX17 (rs1504749 and rs1958409) in Europeans was different with that in East Asian populations (Supplementary Fig. 1, only online), which illustrated that rs10958409 near SOX17 might be a specific variant for IA in the European population.

$R B B P 8$ is located at chromosome 18q11.2 and may be a tumor suppressor. ${ }^{39}$ Conflicting results have been reported for $R B B P 8$ variants in relation to IA in previous GWASs. Yasuno, et al. ${ }^{10}$ reported that an allele of rs11661542 was significantly associated with IA in Europeans. However, the association was not replicated in a Japanese population. ${ }^{14}$ Hong, et al ${ }^{37}$ reported that rs11661542 had no effect on IA in a Korean population. Our results showed that rs11661542 was not associated with IA in Chinese under any genetic model, which was consistent with the findings in Korea and Japan. The conflicting results between European and Asian populations may be due to population heterogeneity. According to the HapMap database, the minor allele frequency of rs 11661542 was 0.44 in Europeans, which was higher than that in Asians (Japanese: 0.37; Korean: 0.37; Chinese: 0.38, respectively). ${ }^{10,14,37}$ Therefore, differential allele frequencies of rs11661542 might have different influences on IA risk in European and Asian populations.

The limitations of this study should be mentioned. First, due to the invasive examination and relative expensive cost of cerebrovascular disease checkup, definitely IA-free controls were not available, and we used individuals who did not have medical (or family) history of IA and other cerebrovascular diseases as controls. These individuals were recruited from the community who were attending a routine annual health check-up during the same period and were relatively older than the IA cases. This may perturb the association results. However, considering that the individuals in the control group were without IA and that they should also have been healthy 10 years prior, the results could not be perturbed significantly. Additionally, further logistic regression analysis was performed using additional controls (208 Chinese Han individuals from the 1000 Genome Project), and the results mostly consistent with our current findings (Supplementary Table 2, only online), illustrating that our results are stable. Second, we did not perform functional studies, and the exact mechanisms of these variants on IA remain unclear. Further experimental studies are needed. Third, in this study, we only considered variants identified in GWAS, and more comprehensive studies need to be considered in future etiology studies. Recently, exome sequencing has been used to identify rare variants in several 
genes significantly associated with IA risk in familial cases. ${ }^{40}$ Whether rare variants of these genes are reflective of the susceptibility to sporadic IA needs to be explored.

In conclusion, we confirmed associations between common variants in $C D K N 2 B-A S 1, R P 1$, and $H D A C 9$ and IA in a Chinese population. Our study provides information for a better understanding of IA pathogenesis, although further studies are needed to explore the potential mechanisms of theses variants.

\section{ACKNOWLEDGEMENTS}

This work was supported by grants from the National Nature Science Foundation of China, Nature Science Foundation of Hunan province and China postdoctoral science foundation to J.Y (No. 81502881, 2017JJ3428, and 2015M582351). Dr. Yan is a postdoctoral fellow at Central South University (No. 149946), supported by the Postdoctoral International Exchange Plan in China.

We thank all participants for providing samples and also those involved in the sample collection.

\section{AUTHOR CONTRIBUTIONS}

Conceptualization: Bingyang Li, Chongyu Hu, Junxia Yan. Data curation: Bingyang Li. Formal analysis: Bingyang Li. Funding acquisition: Junxia Yan. Investigation: Bingyang Li, Chongyu Hu, Junyu Liu, Xin Liao, Jiayu Xun, Manqian Xiao. Methodology: Bingyang Li, Chongyu $\mathrm{Hu}$, Junxia Yan. Project administration: Bingyang Li, Chongyu Hu, Junxia Yan. Resources: Chongyu Hu, Junyu Liu, Junxia Yan. Supervision: Bingyang Li, Chongyu Hu, Junxia Yan. Writing_original draft: Bingyang Li, Chongyu Hu. Writing_review \& editing: Junxia Yan.

\section{ORCID iDs}

$\begin{array}{ll}\text { Bingyang Li } & \text { https://orcid.org/0000-0003-0138-773X } \\ \text { Chongyu Hu } & \text { https://orcid.org/0000-0002-4086-0916 } \\ \text { Junyu Liu } & \text { https://orcid.org/0000-0002-3803-4068 } \\ \text { Xin Liao } & \text { https://orcid.org/0000-0002-9640-8638 } \\ \text { Jiayu Xun } & \text { https://orcid.org/0000-0002-9583-968X } \\ \text { Manqian Xiao } & \text { https://orcid.org/0000-0002-4844-3412 } \\ \text { Junxia Yan } & \text { https://orcid.org/0000-0001-6067-1613 }\end{array}$

\section{REFERENCES}

1. Vlak MH, Algra A, Brandenburg R, Rinkel GJ. Prevalence of unruptured intracranial aneurysms, with emphasis on sex, age, comorbidity, country, and time period: a systematic review and meta-analysis. Lancet Neurol 2011;10:626-36.

2. Li MH, Chen SW, Li YD, Chen YC, Cheng YS, Hu DJ, et al. Prevalence of unruptured cerebral aneurysms in Chinese adults aged 35 to 75 years: a cross-sectional study. Ann Intern Med 2013;159: 514-21.

3. Hitchcock E, Gibson WT. A review of the genetics of intracranial berry aneurysms and implications for genetic counseling. J Genet Couns 2017;26:21-31.

4. Darsaut TE, Findlay JM, Magro E, Kotowski M, Roy D, Weill A, et al. Surgical clipping or endovascular coiling for unruptured intracranial aneurysms: a pragmatic randomised trial. J Neurol Neurosurg Psychiatry 2017;88:663-8.

5. Backes D, Rinkel GJ, Laban KG, Algra A, Vergouwen MD. Patientand aneurysm-specific risk factors for intracranial aneurysm growth: a systematic review and meta-analysis. Stroke 2016;47: 951-7.

6. Bourcier R, Redon R, Desal H. Genetic investigations on intracranial aneurysm: update and perspectives. J Neuroradiol 2015;42: 67-71.

7. Tromp G, Weinsheimer S, Ronkainen A, Kuivaniemi H. Molecular basis and genetic predisposition to intracranial aneurysm. Ann Med 2014;46:597-606.

8. Alg VS, Sofat R, Houlden H, Werring DJ. Genetic risk factors for intracranial aneurysms: a meta-analysis in more than 116,000 individuals. Neurology 2013;80:2154-65.

9. Yasuno K, Bakırcıoğlu M, Low SK, Bilgüvar K, Gaál E, Ruigrok YM, et al. Common variant near the endothelin receptor type A (EDNRA) gene is associated with intracranial aneurysm risk. Proc Natl Acad Sci U S A 2011;108:19707-12.

10. Yasuno K, Bilguvar K, Bijlenga P, Low SK, Krischek B, Auburger G, et al. Genome-wide association study of intracranial aneurysm identifies three new risk loci. Nat Genet 2010;42:420-5.

11. Hashikata H, Liu W, Inoue K, Mineharu Y, Yamada S, Nanayakkara $\mathrm{S}$, et al. Confirmation of an association of single-nucleotide polymorphism rs1333040 on 9p21 with familial and sporadic intracranial aneurysms in Japanese patients. Stroke 2010;41:1138-44.

12. Liu L, Huang X, Cai Y, Feng K, DU J, Lu H, et al. [Correlation between endothelin receptor type A gene polymorphism and sporadic intracranial aneurysms]. Nan Fang Yi Ke Da Xue Xue Bao 2014;34:60-4.

13. Chen Y, Li G, Fan H, Guo S, Li R, Yin J, et al. CDKN2BAS gene polymorphisms and the risk of intracranial aneurysm in the Chinese population. BMC Neurol 2017;17:214.

14. Low SK, Takahashi A, Cha PC, Zembutsu H, Kamatani N, Kubo M, et al. Genome-wide association study for intracranial aneurysm in the Japanese population identifies three candidate susceptible loci and a functional genetic variant at EDNRA. Hum Mol Genet 2012;21:2102-10.

15. Bilguvar K, Yasuno K, Niemelä M, Ruigrok YM, von Und Zu Fraunberg M, van Duijn CM, et al. Susceptibility loci for intracranial aneurysm in European and Japanese populations. Nat Genet 2008; 40:1472-7.

16. Foroud T, Koller DL, Lai D, Sauerbeck L, Anderson C, Ko N, et al. Genome-wide association study of intracranial aneurysms confirms role of Anril and SOX17 in disease risk. Stroke 2012;43:2846-52.

17. Foroud T, Lai D, Koller D, Van't Hof F, Kurki MI, Anderson CS, et al. Genome-wide association study of intracranial aneurysm identifies a new association on chromosome 7. Stroke 2014;45:3194-9.

18. Kurki MI, Gaál EI, Kettunen J, Lappalainen T, Menelaou A, Anttila V, et al. High risk population isolate reveals low frequency variants predisposing to intracranial aneurysms. PLoS Genet 2014;10: e1004134.

19. Barrett JC, Fry B, Maller J, Daly MJ. Haploview: analysis and visualization of LD and haplotype maps. Bioinformatics 2005;21:263-5.

20. Mueller JC. Linkage disequilibrium for different scales and applications. Brief Bioinform 2004;5:355-64.

21. Olsson S, Csajbok LZ, Jood K, Nylén K, Nellgård B, Jern C. Association between genetic variation on chromosome 9p21 and aneurysmal subarachnoid haemorrhage. J Neurol Neurosurg Psychiatry 2011;82:384-8.

22. Zholdybayeva EV, Medetov YZ, Aitkulova AM, Makhambetov YT, Akshulakov SK, Kaliyev AB, et al. Genetic risk factors for intracra- 
nial aneurysm in the Kazakh population. J Mol Neurosci 2018;66: 135-45.

23. Abrantes P, Santos MM, Sousa I, Xavier JM, Francisco V, Krug T, et al. Genetic variants underlying risk of intracranial aneurysms: insights from a GWAS in Portugal. PLoS One 2015;10:e0133422.

24. Nakaoka H, Takahashi T, Akiyama K, Cui T, Tajima A, Krischek B, et al. Differential effects of chromosome 9p21 variation on subphenotypes of intracranial aneurysm: site distribution. Stroke 2010;41:1593-8.

25. Deka R, Koller DL, Lai D, Indugula SR, Sun G, Woo D, et al. The relationship between smoking and replicated sequence variants on chromosomes 8 and 9 with familial intracranial aneurysm. Stroke 2010;41:1132-7.

26. Visel A, Zhu Y, May D, Afzal V, Gong E, Attanasio C, et al. Targeted deletion of the 9p21 non-coding coronary artery disease risk interval in mice. Nature 2010;464:409-12.

27. Jarinova O, Stewart AF, Roberts R, Wells G, Lau P, Naing T, et al. Functional analysis of the chromosome 9p21.3 coronary artery disease risk locus. Arterioscler Thromb Vasc Biol 2009;29:1671-7.

28. Burd CE, Jeck WR, Liu Y, Sanoff HK, Wang Z, Sharpless NE. Expression of linear and novel circular forms of an INK4/ARF-associated non-coding RNA correlates with atherosclerosis risk. PLoS Genet 2010;6:e1001233.

29. Kabir F, Ullah I, Ali S, Gottsch AD, Naeem MA, Assir MZ, et al. Loss of function mutations in RP1 are responsible for retinitis pigmentosa in consanguineous familial cases. Mol Vis 2016;22:610-25.

30. Hong EP, Kim BJ, Kim C, Choi HJ, Jeon JP. Association of SOX17 gene polymorphisms and intracranial aneurysm: a case-control study and meta-analysis. World Neurosurg 2018;110:e823-9.

31. Han X, Han X, Wang Z, Shen J, Dong Q. HDAC9 regulates ox-LDLinduced endothelial cell apoptosis by participating in inflammatory reactions. Front Biosci (Landmark Ed) 2016;21:907-17.
32. Ding H, Xu Y, Bao X, Wang X, Cui G, Wang W, et al. Confirmation of genomewide association signals in Chinese Han population reveals risk loci for ischemic stroke. Stroke 2010;41:177-80.

33. Traylor M, Farrall M, Holliday EG, Sudlow C, Hopewell JC, Cheng YC, et al. Genetic risk factors for ischaemic stroke and its subtypes (the METASTROKE collaboration): a meta-analysis of genomewide association studies. Lancet Neurol 2012;11:951-62.

34. Dichgans M, Malik R, König IR, Rosand J, Clarke R, Gretarsdottir $\mathrm{S}$, et al. Shared genetic susceptibility to ischemic stroke and coronary artery disease: a genome-wide analysis of common variants. Stroke 2014;45:24-36.

35. Shroff N, Ander BP, Zhan X, Stamova B, Liu D, Hull H, et al. HDAC9 polymorphism alters blood gene expression in patients with large vessel atherosclerotic stroke. Transl Stroke Res 2019; 10:19-25.

36. Mikhail M, Vachon PH, D'Orléans-Juste P, Jacques D, Bkaily G. Role of endothelin-1 and its receptors, ETA and ETB, in the survival of human vascular endothelial cells. Can J Physiol Pharmacol 2017; 95:1298-305.

37. Hong EP, Kim BJ, Cho SS, Yang JS, Choi HJ, Kang SH, et al. Genomic variations in susceptibility to intracranial aneurysm in the Korean population. J Clin Med 2019;8:E275.

38. Hirate Y, Suzuki H, Kawasumi M, Takase HM, Igarashi H, Naquet $P$, et al. Mouse Sox17 haploinsufficiency leads to female subfertility due to impaired implantation. Sci Rep 2016;6:24171.

39. Miao WJ, Yuan DJ, Zhang GZ, Liu Q, Ma HM, Jin QQ. IncRNA CASC2/miR18a5p axis regulates the malignant potential of nasopharyngeal carcinoma by targeting RBBP8. Oncol Rep 2019;41: 1797-806.

40. Zhou S, Dion PA, Rouleau GA. Genetics of intracranial aneurysms. Stroke 2018;49:780-7. 